\title{
Comparative Evaluation of Three Homogenization Methods for Isolating Middle East Respiratory Syndrome Coronavirus Nucleic Acids From Sputum Samples for Real-Time Reverse Transcription PCR
}

\author{
Heungsup Sung, M.D. ${ }^{1}$, Dongeun Yong, M.D. ${ }^{2}$, Chang-Seok Ki, M.D. ${ }^{3}$, Jae-Seok Kim, M.D. ${ }^{4}$, Moon-Woo Seong, M.D. ${ }^{5}$, \\ Hyukmin Lee, M.D. ${ }^{6}$, and Mi-Na Kim, M.D. ${ }^{1}$ \\ Department of Laboratory Medicine ${ }^{1}$, University of Ulsan College of Medicine and Asan Medical Center, Seoul; Department of Laboratory Medicine², \\ Severance Hospital, Yonsei University College of Medicine, Seoul; Department of Laboratory Medicine and Genetics ${ }^{3}$, Samsung Medical Center, \\ Sungkyunkwan University School of Medicine, Seoul; Department of Laboratory Medicine ${ }^{4}$, Hallym University College of Medicine, Hallym University \\ Kangdong Sacred Heart Hospital, Seoul; Department of Laboratory Medicine ${ }^{5}$, Seoul National University College of Medicine and Seoul National University \\ Hospital, Seoul; Department of Laboratory Medicine ${ }^{6}$, International St. Mary's Hospital, Catholic Kwandong University College of Medicine, Incheon, Korea
}

Background: Real-time reverse transcription PCR (rRT-PCR) of sputum samples is commonly used to diagnose Middle East respiratory syndrome coronavirus (MERS-CoV) infection. Owing to the difficulty of extracting RNA from sputum containing mucus, sputum homogenization is desirable prior to nucleic acid isolation. We determined optimal homogenization methods for isolating viral nucleic acids from sputum.

Methods: We evaluated the following three sputum-homogenization methods: proteinase $\mathrm{K}$ and DNase I (PK-DNase) treatment, phosphate-buffered saline (PBS) treatment, and $\mathrm{N}$ acetyl-L-cysteine and sodium citrate (NALC) treatment. Sputum samples were spiked with inactivated MERS-CoV culture isolates. RNA was extracted from pretreated, spiked samples using the easyMAG system (bioMérieux, France). Extracted RNAs were then subjected to rRT-PCR for MERS-CoV diagnosis (DiaPlex Q MERS-coronavirus, SolGent, Korea).

Results: While analyzing 15 spiked sputum samples prepared in technical duplicate, false-negative results were obtained with five (16.7\%) and four samples (13.3\%), respectively, by using the PBS and NALC methods. The range of threshold cycle (Ct) values observed when detecting upE in sputum samples was 31.1-35.4 with the PK-DNase method, 34.7-39.0 with the PBS method, and 33.9-38.6 with the NALC method. Compared with the control, which were prepared by adding a one-tenth volume of 1:1,000 diluted viral culture to PBS solution, the ranges of Ct values obtained by the PBS and NALC methods differed significantly from the mean control Ct of 33.2 (both $P<0.0001$ ).

Conclusions: The PK-DNase method is suitable for homogenizing sputum samples prior to RNA extraction.

Key Words: MERS coronavirus, Sputum, Homogenization, Nucleic acid extraction, Comparison, Proteinase K, DNase
Received: September 17, 2015

Revision received: March 22, 2016

Accepted: May 17, 2016

Corresponding author: Heungsup Sung Department of Laboratory Medicine, University of Ulsan College of Medicine and Asan Medical Center, 88 Olympic-ro 43-gil, Songpa-gu, Seoul 05505, Korea

Tel: +82-2-3010-4499

Fax: +82-2-478-0884

E-mail: sung@amc.seoul.kr

Co-corresponding author: Mi-Na Kim Department of Laboratory Medicine, University of Ulsan College of Medicine and Asan Medical Center, 88 Olympic-ro 43-gil, Songpa-gu, Seoul 05505, Korea

Tel: +82-2-3010-4511

Fax: +82-2-478-0884

E-mail:mnkim@amc.seoul.kr

\section{INTRODUCTION}

Rapid and accurate diagnosis of infection and control measures are critical for preventing the spread of Middle East respiratory syndrome coronavirus (MERS-CoV) [1]. For laboratory diagnosis of MERS-CoV infection, lower respiratory samples are preferred 
irrespective of the length of time between symptom onset and sample collection [2]. Real-time reverse transcription PCR (rRTPCR) with sputum samples is commonly used to diagnose MERS-CoV infection.

If a negative result is obtained for patients with a high index of suspicion for MERS-CoV infection, additional samples should be collected and tested [3]. A series of negative results does not rule out the possibility of MERS-CoV infection [1, 3]. Possible explanations for false-negative MERS-CoV test results include low viral load very early or late in the illness, inadequate sputum samples due to poor sample collection from patients with dry cough or no cough, inappropriate sample handling or shipping, and technical issues, such as viral genome mutation or PCR inhibition [3]. Sufficient nucleic acid extraction and the removal of substances that inhibit amplification are critical factors influencing the detection of MERS-CoV by rRT-PCR [4]. However, rRTPCR of sputum samples yields conflicting results owing to the difficulty of extracting RNA from sputum containing mucus. Many reports have indicated that lower respiratory samples such as bronchoalveolar lavage (BAL) fluid and sputum contain the highest viral loads [3, 5-7]. However, one report indicated that no significant difference occurred in viral load cycle threshold (Ct) values between sputa and nasopharyngeal aspirates (NPAs) [8]. For this reason, sputum sample homogenization is desirable prior to isolating nucleic acids for MERS-CoV rRT-PCR. In this study, we compared three different sputum homogenization methods and identified an optimal homogenization method prior to the nucleic acid isolation from sputum samples.

\section{METHODS}

\section{Sputum quality}

Forty-eight consecutive sputum samples sent to the laboratory for MERS-CoV rRT-PCR testing were evaluated in terms of the number of white blood cells, squamous epithelial cells, and ciliated columnar epithelial cells. The sputum samples were graded by using Murray and Washington's grading system [9].

\section{Preparation of virus-spiked sputum samples}

An inactivated viral culture of MERS-CoV was provided by the Division of Respiratory Viruses (Center for Infectious Diseases, Korean National Institute of Health, Korean Center for Disease Control). The Korean National Institute of Health did not disclose the exact titer of the cultured MERS-CoV. Since other viral respiratory pathogens and bacterial pathogens should be considered during diagnosis [2], we evaluated whether the three homogenization methods have an adverse impact on the detection of other respiratory viruses. For this reason, we evaluated three homogenization methods using NPA samples that were found to contain various respiratory viruses using the Anyplex II RV16 Detection kit (Seegene, Seoul, Korea). Next, we evaluated the three homogenization methods using sputum samples spiked with a 1:10 or 1:1,000 dilution of viral culture mixed with an identical volume of sputum. Finally, we evaluated each homogenization method using virus-spiked sputum samples prepared by adding one volume of 1:100 diluted inactivated viral culture to nine volumes of clinical sample (i.e., sputum or BAL).

\section{Sputum-homogenization methods}

Sputum samples were pretreated by using one of three methods. The first method involved proteinase $\mathrm{K}$ and DNase I treatment (PK-DNase), as recommended by the NucliSENS easyMAG protocol (bioMérieux SA, Marcy-l'Etoile, France). One hundred microliters of proteinase $\mathrm{K}$ (1 $\mathrm{mg}$ proteinase $\mathrm{K} / 1 \mathrm{~mL}$ proteinase $\mathrm{K}$ buffer; Promega, Madison, WI, USA) was added to $100 \mu \mathrm{L}$ of sample and incubated for $15 \mathrm{~min}$ at $55^{\circ} \mathrm{C}$. The samples were mixed by vortexing every 5 min. Subsequently, a 20$\mu \mathrm{L}$ of DNase I solution $(1 \mathrm{U} / \mu \mathrm{L})$ was added for every $100 \mu \mathrm{L}$ of sample, followed by incubation at $37^{\circ} \mathrm{C}$ for $30 \mathrm{~min}$. In the second method, sputum samples were mixed with an equal volume of phosphate-buffered saline (PBS). In the third method, the sputum samples were mixed with an equal volume of $\mathrm{N}$-acetylL-cysteine (NALC) in sodium citrate solution (prepared by dissolving $0.5 \mathrm{~g}$ NALC in each $100 \mathrm{~mL}$ of $1.47 \%$ sodium citrate solution). Following the second and third methods, the samples were mixed by vortexing for 1 min.

\section{RNA extraction and MERS-CoV rRT-PCR testing}

RNA was extracted as described previously [10] by using the NucliSENS easyMAG system (bioMérieux SA, Marcy-l'Etoile, France). RNA from each pretreated sample $(500 \mu \mathrm{L})$ was eluted in a $50-\mu \mathrm{L}$ volume. Extracted RNA was tested by rRT-PCR using Anyplex II RV16 Detection kit (Seegene) for the preliminary test, or by rRT-PCR using DiaPlexQ MERS Virus Detection kit (SolGent, Daejeon, Korea) with Bio-Rad CFX96 instrument (BioRad, Hercules, CA, USA). The sequences of the primers and probes used with the DiaPlexQ kit covered the same general regions of the upE and ORFla transcripts.

\section{Statistical analysis}

Quantitative results were expressed as mean $\pm S D$, and groups were compared by using the one-sample t-test. $P$ values $<0.05$ 
were considered statistically significant. MedCalc software version 16.2 (MedCalc Software bvba, Ostend, Belgium) was used for statistical analysis.

\section{RESULTS}

According to Murray and Washington's grading system [9], among the 48 sputum samples tested, 18 (37.5\%) belonged to group 1, 6 (12.5\%) belonged to group 3, 2 (4.2\%) belonged to group 4, 5 (10.4\%) belonged to group 5, and 17 (35.4\%) belonged to group 6 . Thirty-two samples (66.7\%), including 20 from groups 1-3, harbored a detectable quantity of ciliated columnar epithelial cells. Seventeen samples (35.4\%) harbored identifiable proteinaceous mucous threads (viewed via light microscopy).

The efficacies of the three homogenization methods tested with NPA samples are summarized in Table 1. For DNA respiratory viruses (such as adenovirus and human bocavirus), PKDNase treatment caused a slight increase in Ct values compared with PBS or NALC treatment. When we additionally tested six adenovirus-positive NPAs and five bocavirus-positive NPAs, the Ct values obtained following PBS preparation were 1.506.18 (median 5.42) and 1.60-7.61 (median 3.4) lower, respectively, than those obtained following PK-DNase preparation. Among the 10 samples derived from RNA respiratory viruses, the minimum Ct values resulted from PK-DNase treatment of three samples, PBS treatment of five samples, and NALC treatment of two samples.

Using identical volumes of sputum and inactivated viral culture diluted 1:10 or 1:1,000, the effects of each homogenization method on the MERS-CoV rRT-PCR results were evaluated (Table 2). Using a 1:10 dilution of inactivated viral culture, all samples treated with PK-DNase exhibited lower Ct values than those subjected to PBS or NALC treatment. The differences in Ct values between samples treated with PK-DNase or PBS were 5.47.8 for upE and 4.4-6.8 for ORF1a, while those for samples treated with PK-DNase or NALC were 4.7-5.8 for upE and 4.45.6 for ORF1a. Using the same volume of a 1:1,000 dilution of inactivated viral culture, one purulent sputum sample showed a false-negative result after PBS treatment. The $\mathrm{Ct}$ values of the other two sputum samples were similar following PK-DNase, PBS, and NALC treatments.

Using a one-tenth volume of a 1:1,000 dilution of inactivated viral culture (to simulate conditions comparable to those of real clinical samples), the effects of each method on the MERS-CoV rRT-PCR results were evaluated (Table 3). Among the three sputum samples, false-negative results were detected in two samples following PBS treatment and in three samples after NALC treatment (Table 3). Although the Ct values after PK-DNase treatment tended to be lower than those following PBS or

Table 1. Cycle threshold (Ct) values following three homogenization methods used before Anyplex II RV16 detection of respiratory viruses in nasopharyngeal aspirates by rRT-PCR

\begin{tabular}{lcccccccc}
\hline \multirow{2}{*}{ Homogenization by } & \multicolumn{7}{c}{ Respiratory virus (N) } \\
\cline { 2 - 8 } & ADV (1) & BoV (2) & INF A (2) & RhinoV (1) & CoV 229E/NL63 (2) & CoV 0C43 (2) & RSV (2) & hMPV (1) \\
\hline PK-DNase I & 35.6 & $26.5 / 29.9$ & $20.8 / 42.6$ & 33.4 & $31.2 / 42.7$ & $32.7 / 37.4$ & $35.8 / 40.4$ & 42.2 \\
PBS & 34.1 & $21.6 / 28.3$ & $19.8 / 41.9$ & 31.7 & $33.2 / 44.3$ & $35.8 / 35.4$ & $37.0 / 37.3$ & 38.3 \\
NALC-sodium citrate & 33.1 & $22.4 / 28.6$ & $21.1 / 42.0$ & 32.3 & $30.8 / 45.1$ & $35.0 / 35.6$ & $36.3 / 35.1$ & 39.2 \\
\hline
\end{tabular}

Abbreviations: rRT-PCR, Real-time reverse transcription PCR; PK, proteinase K; PBS, phosphate-buffered saline; NALC, N-acetyl-L-cysteine; ADV, adenovirus; BoV, human bocavirus; INF A, influenza virus A; RhinoV, rhinovirus; CoV, human coronavirus; RSV, respiratory syncytial virus; hMPV, human metapneumovirus.

Table 2. Cycle threshold (Ct) values observed with three homogenization methods following mixture of identical volumes of a $1: 10$ or 1:1,000 dilution of inactivated MERS-CoV culture with sputum samples

\begin{tabular}{|c|c|c|c|c|c|}
\hline \multirow{2}{*}{ Homogenization by } & \multirow{2}{*}{$\mathrm{N}$} & \multicolumn{2}{|c|}{ 1:10 diluted cell culture } & \multicolumn{2}{|c|}{ 1:1,000 diluted cell culture } \\
\hline & & upE & ORF1a & upE & ORF1a \\
\hline PK-DNase 1 & 3 & $17.6 / 20.9 / 20.2^{*}$ & 18.1/21.1/20.4 & $34.2 / 34.6 / 34.3$ & $35.7 / 35.6 / 35.8$ \\
\hline PBS & 3 & $23.4 / 26.3 / 28.0$ & $22.5 / 26.1 / 27.2$ & 34.6/34.4/Neg & 35.3/35.8/Neg \\
\hline NALC-sodium citrate & 3 & 23.0/25.6/26.0 & $22.5 / 25.6 / 26.0$ & $35.6 / 33.0 / 34.9$ & $37.6 / 34.1 / 36.1$ \\
\hline
\end{tabular}

*The 3 sputum samples shown include 1 saliva-like sputum sample, 1 non-purulent sputum sample, and 1 purulent sputum sample, with the results shown in this order.

Abbreviations: NALC, N-acetyl-L-cysteine; Neg, negative; PBS, phosphate-buffered saline; PK, proteinase K. 
Table 3. Cycle threshold (Ct) values for triplicate sputum and bronchoalveolar lavage (BAL) samples following processing using three different homogenization methods

\begin{tabular}{|c|c|c|c|c|c|}
\hline \multirow{2}{*}{ Homogenization by } & \multirow{2}{*}{$N^{*}$} & \multicolumn{2}{|c|}{ Sputum } & \multicolumn{2}{|c|}{ BAL } \\
\hline & & upE & ORF1a & upE & ORFla \\
\hline PK-DNase 1 & 3 & $34.9 / 36.3 / 34.0$ & $35.0 / 34.9 / 35.4$ & 34.1/33.9/34.4 & $34.6 / 34.0 / 35.1$ \\
\hline PBS & 3 & $\mathrm{Neg} / 37.9 / \mathrm{Neg}$ & $\mathrm{Neg} / \mathrm{Neg} / \mathrm{Neg}$ & $34.1 / 33.9 / 33.7$ & $34.8 / 34.4 / 35.0$ \\
\hline NALC-sodium citrate & 3 & Neg/Neg/Neg & Neg/Neg/Neg & $35.0 / 34.6 / 34.3$ & $35.7 / 35.8 / 35.5$ \\
\hline
\end{tabular}

*Three different sputum and bronchoalveolar lavage samples were included, and results are provided in this order.

Abbreviations: NALC, N-acetyl-L-cysteine; Neg, negative; PBS, phosphate-buffered saline; PK, proteinase K.

Table 4. Cycle threshold (Ct) values for upE measured with duplicate sputum samples following processing using three homogenization methods

\begin{tabular}{lcccc}
\hline Homogenization by & Sample N (Results N) & N of positive results (\%) & $\begin{array}{c}\text { Ct value from positive } \\
\text { samples, mean } \pm \text { SD (range) }\end{array}$ & $P_{\text {value* }}$ \\
\hline PK-DNase 1 & $15(30)$ & $30(100)$ & $33.6 \pm 1.3(31.1-35.4)$ & 0.11 \\
PBS & $15(30)$ & $25(83.3)$ & $35.8 \pm 1.2(34.7-39.0)$ & $<0.0001$ \\
NALC-sodium citrate & $15(30)$ & $26(86.7)$ & $35.7 \pm 1.5(33.9-38.6)$ & $<0.0001$ \\
\hline
\end{tabular}

*The significance level was calculated by using the one-sample t-test. The null hypothesis was that the test mean was equal to the control mean of 33.2 . Abbreviations: NALC, N-acetyl-L-cysteine; PBS, phosphate-buffered saline; PK, proteinase K.

NALC treatment, the values for BAL samples did not differ markedly depending on the homogenization method used.

To assess statistical significance, we tested 15 additional spiked samples, which were prepared by adding a one-tenth volume of 1:1,000 diluted viral culture (Table 4). Among the 15 spiked sputum samples prepared in duplicate (30 total samples), false-negative results were detected in five samples (16.7\%) using the PBS method and in four samples (13.3\%) using the NALC method. The ranges of $\mathrm{Ct}$ values for upE from sputum samples were 31.1-35.4 using the PK-DNase method, 34.7-39.0 using the PBS method, and 33.9-38.6 using the NALC method. Compared with the control, which were prepared by adding a one-tenth volume of 1:1,000 diluted viral culture to PBS solution, the ranges of $\mathrm{Ct}$ values following the PBS and NALC methods significantly differed from the mean Ct value (33.2) of the control (both $P<0.0001$ ).

\section{DISCUSSION}

For the extraction of MERS-CoV RNA from sputum sample, homogenization of sputum by PK-DNase I treatment was superior to treatment with PBS or NALC and sodium citrate. Proteinase $\mathrm{K}$ is a serine protease that exhibits broad cleavage specificity [11]. The enzyme cleaves peptide bonds adjacent to carboxylic group of aliphatic and aromatic amino acids and is useful for the general digestion of proteins in biological samples. More- over, proteinase $\mathrm{K}$ degrades any RNases present in samples, preventing RNA degradation. Viral nucleic acids are protected from DNase I degradation by the viral protein capsids, although some enveloped viruses may be lost [12]. The genomes of DNA viruses such as adenovirus and bocavirus can be affected by proteinase $\mathrm{K}$ and DNase I, possibly owing to the digestion of viral capsid proteins by proteinase $\mathrm{K}$ and subsequent degradation of genomic DNA by DNase I.

Confirmation of probable or suspected MERS-CoV infection based on clinical and epidemiological criteria can only be accomplished through laboratory testing. However, other viral respiratory pathogens and bacterial pathogens should be considered during diagnosis [3]. When using lower respiratory samples originally earmarked for MERS-CoV testing, other viral respiratory pathogens should be tested for routine diagnostic processes. Because the ability to detect DNA respiratory viruses can be reduced by DNase I treatment, proteinase $\mathrm{K}$ treatment alone is worth considering for the routine detection of respiratory viruses from sputum samples.

The United States Centers for Disease Control and Prevention recommends that respiratory samples be extracted by using either the NucliSENS easyMAG system or MagNA Pure Compact Nucleic Acid Isolation kit [2]. Sputum samples categorized into groups 4-5 according to Murray and Washington's grading system [9] usually cannot undergo automated extraction owing to high viscosity and, thus, require a pre-homogenization process. 
Treatment with PBS or NALC and sodium citrate occasionally produces false-negative results with purulent sputum. Adding PBS and mixing via vortexing are necessary to culture respiratory viruses from sputum, and this procedure offers the advantage of involving minimum sample handling. However, thoroughly homogenizing thick or purulent sputum using this method is problematic. Because viral isolation by cell culture is not recommended for the routine confirmation of cases of MERS-CoV [2, 3], the PBS plus vortexing method for processing sputum samples is not suitable for detecting MERS-CoV by rRTPCR. Previously, it was reported that NALC and sodium citrate treatment (without $\mathrm{NaOH}$ ) did not damage mycobacterial mRNA or rRNA in sputum samples [13]. NALC and sodium citrate solutions are readily available in clinical microbiology laboratories. Treatment with NALC and sodium citrate solution resulted in complete homogenization of sputum samples, including purulent sputum. However, this method is not suitable for detecting RNA targets in purulent sputum. Because proteinase $\mathrm{K}$ treatment requires heat inactivation of potential pathogens (incubation for $15 \mathrm{~min}$ at $55^{\circ} \mathrm{C}$ ), this method offers additional laboratory safety.

Only $10 \%$ of sputum samples sent for MERS-CoV rRT-PCR testing were clinically relevant in terms of bacterial culture (group 5 according to Murray and Washington's grading system [9]). Among patients infected with MERS-CoV, a dry cough is more common than a productive cough [16, 17]. Therefore, optimum sample collection is not feasible for patients unable to produce sputum. However, two thirds of sputum samples in our study contained identifiable ciliated columnar epithelial cells. Dipeptidyl peptidase 4, the human receptor for MERS-CoV [16], is expressed in type I and II alveolar cells, ciliated or non-ciliated bronchial epithelial cells, and bronchial submucosal glands [18, 19]. Although it is difficult to obtain high-quality sputum from patients with no cough or a dry cough, obtaining and testing sputum samples containing ciliated columnar epithelial cells is recommended, if possible.

Our study has several limitations. First, it was performed by using spiked sputum samples; thus, our results may differ from those obtained by using authentic MERS-CoV-positive samples. However, the spiked sputum samples were similar to real sputum samples, as they were produced by adding a one-tenth volume of spiked viral solution to total sputum samples. Second, we tested a relatively small number of sputum samples under each test condition. Therefore, our findings should be confirmed by additional, larger studies.

In conclusion, the PK-DNase I treatment is suitable for the homogenization of sputum prior to RNA extraction for MERSCoV rRT-PCR detection. Furthermore, adequate sputum homogenization prior to the isolation of nucleic acids is critical for accurate diagnosis of MERS-CoV infection.

\section{Authors' Disclosures of Potential Conflicts of Interest}

No potential conflicts of interest relevant to this article were reported.

\section{Acknowledgments}

This work was supported by the Korean Center for Disease Control and the BioNano Health-Guard Research Center, funded by the Ministry of Science, ICT \& Future Planning (MSIP) of Korea as a Global Frontier Project (Grant Number H-GUARD_ERND2-5).

\section{REFERENCES}

1. Lee JH, Lee CS, Lee HB. An appropriate lower respiratory tract sample is essential for diagnosis of Middle East Respiratory Syndrome (MERS). J Korean Med Sci 2015;30:1207-8.

2. Centers for Disease Control and Prevention. Interim guidelines for collecting, handling, and testing clinical specimens from patients under investigation (PUIs) for Middle East Respiratory Syndrome Coronavirus (MERS-CoV)-Version 2.1. http://www.cdc.gov.coronavirus/mers/downloads/guidelines-clinical-specimens.pdf (Updated on Jun 2015).

3. Centers for Disease Control and Prevention. Middle East Respiratory Syndrome (MERS). http://www.cdc.gov/coronavirus/mers/interim-guidance.html. (Last visited on 6 Jul 2015).

4. Wilson D, Yen-Lieberman B, Reischl U, Warshawsky I, Procop GW. Comparison of five methods for extraction of Legionella pneumophila from respiratory specimens. J Clin Microbiol 2004;42:5913-6.

5. Guery B, Poissy J, el Mansouf L, Séjourné C, Ettahar N, Lemaire X, et al; MERS-CoV study group. Clinical features and viral diagnosis of two cases of infection with Middle East Respiratory Syndrome coronavirus: a report of nosocomial transmission. Lancet 2013;381:2265-72.

6. Kapoor M, Pringle K, Kumar A, Dearth S, Liu L, Lovchik J, et al. Clinical and laboratory findings of the first imported case of Middle East respiratory syndrome coronavirus to the United States. Clin Infect Dis 2014; 59:1511-8.

7. Drosten C, Seilmaier M, Corman VM, Hartmann W, Scheible G, Sack S, et al. Clinical features and virological analysis of a case of Middle East respiratory syndrome coronavirus infection. Lancet Infect Dis 2013;13: 745-51.

8. Memish ZA, Al-Tawfiq JA, Makhdoom HQ, Assiri A, Alhakeem RF, Albarrak $A$, et al. Respiratory tract samples, viral load, and genome fraction yield in patients with Middle East respiratory syndrome. J Infect Dis 2014;210:1590-4.

9. Murray PR and Washington JA. Microscopic and bacteriologic analysis of expectorated sputum. Mayo Clin Proc 1975;50:339-44.

10. Kim HK, Oh SH, Yun KA, Sung H, Kim MN. Comparison of Anyplex II RV16 with the XTAG respiratory viral panel and Seeplex RV15 for detec- 
tion of respiratory viruses. J Clin Microbiol 2013;51:1137-41.

11. Sweeney PJ and Walker JM. Proteinase K (EC 3.4.21.14). In: Burrell MM, ed. Methods in molecular biology, Vol. 16. Enzymes of molecular biology. Totowa, NJ: Humana Press, 1993:305-11.

12. Breitbart $B$ and Rohwer F. Method for discovering novel DNA viruses in blood using viral particle selection and shotgun sequencing. Biotechniques 2005;39:729-36.

13. Desjardin LE, Perkins MD, Teixeira L, Cave MD, Eisenach KD. Alkaline decontamination of sputum specimens adversely affects stability of mycobacterial mRNA. J Clin Microbiol 1996;34:2435-9.

14. Erin EM, Jenkins GR, Kon OM, Zacharasiewicz AS, Nicholson GC, Neighbour $\mathrm{H}$, et al. Optimized dialysis and protease inhibition of sputum dithiothreitol supernatants. Am J Respir Crit Care Med 2008;177: 132-41.

15. Grebski E, Peterson C, Medici TC. Effect of physical and chemical methods of homogenization on inflammatory mediators in sputum of asthma patients. Chest 2001;119:1521-5.

16. Zumla A, Hui DS, Perlman S. Middle East respiratory syndrome. Lancet 2015;386:995-1007.

17. Assiri A, Al-Tawfiq JA, Al-Rabeeah AA, Al-Rabiah FA, Al-Hajjar S, AlBarrak $A$, et al. Epidemiological, demographic, and clinical characteristics of 47 cases of Middle East respiratory syndrome coronavirus disease from Saudi Arabia: a descriptive study. Lancet Infect Dis 2013; 13:752-61.

18. Raj VS, Mou H, Smits SL, Dekkers DH, Müller MA, Dijkman R, et al. Dipeptidyl peptidase 4 is a functional receptor for the emerging human coronavirus-EMC. Nature 2013;495:251-4.

19. Poissy J, Goffard A, Parmentier-Decrucq E, Favory R, Kauv M, Kipnis E, et al; MERS-CoV Biology Group. Kinetics and pattern of viral excretion in biological specimens of two MERS-CoV cases. J Clin Virol 2014;61: 275-8. 